research investment by government.

True, many academics hated the REF, which required them to submit large quantities of information to justify their funding. But an inspection of the REF's outcomes, and of the retrospective reviews of the process by international members of the REF assessment panels (see go.nature.com/q919oe), suggests that it has many strengths.

Take the database of nearly 7,000 case studies of the societal impacts of academic research (see http://impact.ref.ac.uk/casestudies). The diversity of the impacts in terms of (for example) health, sustainability, education and economic growth - in the United Kingdom and beyond - is remarkable and inspiring.

And there is no reason to suppose that identifying these outcomes is the equivalent of the impacts tail wagging the research dog. In the REF, the assessment panels gave the societal case studies a mere $20 \%$ weighting, whereas academic performance had a $65 \%$ weighting. No one could sensibly maintain that the outcomes are necessarily predictable and should be required as a basis for funding in future. What does make sense is that the research community can help to ensure a maximal return on taxpayers' money by becoming aware of impacts pathways, and by broadening its outlook on its roles. As the case studies show, this can happen even in areas of research that are unapologetically fundamental.

A study by the independent consultants RAND Europe estimates that the REF impacts-submissions process cost universities about $\mathfrak{E 5 5}$ million (see go.nature.com/dzwbjn). That may seem to justify the concerns of academics and politicians about the burden. But set against the $£ 1.6$-billion budget that it relates to, one might even describe this $3.4 \%$ overhead as a bargain - especially given that the assessment system may become more efficient, and given the virtues of encouraging such impacts.

Mindful of the burdens, the government is evidently tempted to try to find a cheaper method of assessment of both academic and societal impacts using metrics. As is made clear by the REF panels, by a RAND analysis of the impacts evaluation (go.nature.com/yysa6m) and by an independent assessment of metrics in research (go.nature.com/rfrgql), this temptation should be avoided. Insightful review of both types of outputs is the only way to do justice to them.

The impacts case studies provide welcome ammunition to the case for supporting research in all disciplines, and some government departments have been deploying them in that spirit. Readers who care about UK higher education should give their own responses to the proposals before 15 January 2016, at go.nature.com/13rrtx.

\section{Radio interference}

\section{Conflict at the Arecibo Observatory highlights the need for funders to become more flexible.}

\section{$\mathrm{I}$} n more than 50 years of operation, the Arecibo Observatory has enabled some momentous discoveries. Researchers at the observatory in Puerto Rico found in 1965 that the length of a day on Mercury is more like two Earth months than three. The first binary pulsar, detected at Arecibo in 1974, earned its discoverers the 1993 Nobel Prize in Physics. The observatory's telescope made the first confirmed discovery of extrasolar planets in the early 1990s. And two years ago, astronomers reported using Arecibo to find Leo P, a dwarf galaxy that had gone undetected just 2 megaparsecs from the Milky Way.

Unfortunately, that five-decade streak of discoveries could be near its end. Arecibo is long in the tooth and certainly shows its age, but the problem is not that the observatory has exhausted its scientific potential. Its telescope remains the largest single-dish radio instrument in the world, and is in demand from those who study topics such as pulsars, asteroids and Earth's upper atmosphere.

The problem is money, as it so often is. Operating Arecibo costs about US $\$ 12$ million per year, a price that its owner - the US National Science Foundation (NSF) - feels increasingly unable to pay.

Arecibo's bell began to toll almost a decade ago, when a panel charged with balancing the NSF's astronomy budget put the observatory on its list of potential cuts. The agency's astronomy division has long been under intense pressure to support the development of new instruments such as the Large Synoptic Survey Telescope, currently under construction in Chile, without any increase in budget.

But few in the astronomy community or the NSF seem to relish the idea of closing a facility as scientifically productive as Arecibo. So the agency has endeavoured to find parties willing to contribute to the observatory's operation or, better yet, to take over the enterprise entirely.

In July, a glimmer of hope appeared in the form of the Breakthrough Listen project, a $\$ 100$-million effort sponsored by Internet billionaire Yuri Milner to scan the nearest 1 million stars for signals suggesting the presence of intelligent life. The project has already arranged to purchase about $20 \%$ of the observing time at another NSF radio facility, the Green Bank Telescope in West Virginia.

But no similar agreement has been reached at Arecibo. One

stumbling block may have been conflicts with the observatory's director, Robert Kerr, who has said that the NSF told him that it would cut its contribution to Arecibo by an amount equal to any contribution from Breakthrough Listen. Such an arrangement, he feared, would give the agency an opportunity to absolve itself entirely of the need to fund the telescope (see page 142).

NSF officials vigorously deny any such intent, and insist that they never even suggested a one-for-one reduction of funding as a condition of a Breakthrough Listen deal. Nevertheless, Kerr's objections led to his resignation as operations director. On 26 October, the science agency distributed an open letter soliciting "strategies and goals for continued operations that involve a substantially reduced funding commitment from NSF".

It might seem difficult to understand why the NSF has so much trouble accepting help from a private source.

"Although they may differ on how exactly to proceed, all sides share an interest in the advancement of science." But the agency deserves some sympathy. It has a mandate to support a research agenda dictated by the scientific community through peer review, not by the interests of deeppocketed philanthropists.

That is not to say that Breakthrough Listen's goals lack legitimacy — the argument that intelligent life must exist somewhere else in the universe is strong, the confirmation of a signal from an alien civilization would arguably be the greatest scientific discovery ever made and the Arecibo telescope is one of the world's best instruments for making such a discovery. But accepting Breakthrough Listen's offer without minimizing its impact on the general community's access to the telescope would be irresponsible. The NSF is right to proceed slowly.

Such episodes are likely to be repeated as long as public science funding remains tight and the technology sector continues minting billionaires with the curiosity and intellect to put their money to work expanding the frontiers of human knowledge. In the case of Arecibo, and in future disagreements over how to best combine private and public resources, it is important to remember that although they may differ on how exactly to proceed, all sides share an interest $\rightarrow$ NATURE.COM To comment online, click on Editorials at: go.nature.com/xhunqv in the advancement of science. Elected officials are unlikely to increase funding for research any time soon, so it would be wise for managers at funding agencies and at publicly funded institutions to embrace the spirit of compromise. 